\title{
Incertidumbre ante la enfermedad crónica. Revisión integrativa
}

\author{
Mauricio Arias-Rojas ${ }^{\mathrm{a}}$ - Sonia Carreño Moreno ${ }^{\mathbf{b}}$ - Lorena Chaparro Díaz
}

\begin{abstract}
Resumen: Este estudio tiene como objetivo integrar la evidencia acerca de la incertidumbre ante la enfermedad en el paciente con enfermedad crónica y su cuidador familiar, guiados por la teoría de la incertidumbre ante la enfermedad. Para esto se realizó una síntesis integrativa de la literatura durante los años 2007 a 2017. Se usaron las bases de datos Medline, Science Direct, Ovid Nursing, Scielo, Scopus, cinaHL y Psycinfo en los idiomas inglés y español. Para realizar la síntesis integrativa se incluyeron 46 publicaciones, con 21 estudios de tipo cualitativo, 19 cuantitativo, 2 mixtos y 4 revisiones. Los estudios se realizaron en su mayoría en pacientes con cáncer, enfermedades neurodegenerativas, fallos orgánicos, falla cardiaca, EPOC y en cuidados paliativos de distintas enfermedades. Dentro de las principales conclusiones del estudio se plantea que la persona con enfermedad crónica y su cuidador familiar desarrollan incertidumbre ante la enfermedad crónica debido a falencias en la educación acerca de la enfermedad y el cuidado, así como el soporte social que reciben del equipo de salud y de sus redes de apoyo.
\end{abstract}

Palabras clave: cuidadores; enfermedad crónica; enfermería basada en la evidencia; ética en enfermería; incertidumbre; toma de decisiones

Fecha de Recepción: 18 de julio de 2018

Fecha de Evaluación: 01 de septiembre de 2018

Fecha de aceptación: 26 de noviembre de 2018 Publicación en Línea: 19 de diciembre de 2018

Cómo citar: Arias Rojas, E. M., Carreño Moreno, S. P., \& Chaparro Díaz, O. L. (2019). Incertidumbre ante la enfermedad crónica. Revisión integrativa. Revista Latinoamericana de Bioética, 19(36-1). https://doi.org/10.18359/rlbi.3575

a Doctorando en Enfermería. Becario Colciencias convocatoria 727. Enfermero. Profesor asistente, Facultad de Enfermería, Universidad de Antioquia. Medellín, Colombia.

Correo electrónico: emauricio.arias@udea.edu.co

ORCID: https://orcid.org/0000-0003-2096-1792

b Doctora en Enfermería. Enfermera. Profesora asistente, Facultad de Enfermería, Universidad Nacional de Colombia. Bogotá, Colombia.

Correo electrónico: spcarrenom@unal.edu.co

ORCID: https://orcid.org/0000-0002-4386-6053

c Ec. Doctora en Enfermería. Enfermera. Profesora asociada, Facultad de Enfermería, Universidad Nacional de Colombia. Bogotá, Colombia.

Correo electrónico: olchaparrod@unal.edu.co

ORCID: https://orcid.org/0000-0001-8241-8694 


\section{Uncertainty about Chronic Disease. Integrative Review}

Abstract: This study aims to integrate the evidence about the uncertainty about the disease in the patient with chronic illness and their family caretaker, guided by the theory of uncertainty about the disease. For this, an integrative synthesis of the literature was carried out during the years 2007 to 2017. The Medline, Science Direct, Ovid Nursing, Scielo, Scopus, CINAHL and Psycinfo databases were used in the English and Spanish languages. To carry out the integrative synthesis, 46 publications were included, with 21 qualitative, 19 quantitative, 2 mixed and 4 reviews. The studies were mostly carried out in patients with cancer, neurodegenerative diseases, organic failures, heart failure, COPD and in palliative care of different diseases. Among the main conclusions of the study, it is suggested that the person with chronic illness and their family caretaker develop uncertainty about the chronic illness due to shortcomings in education about the disease and care, as well as the social support they receive from the health team and its support networks.

Keywords: caretakers; chronic illness; nursery based on evidence; nursing ethics; uncertainty; decision making

\section{Incerteza na doença crônica. Revisão integrativa}

Resumo: Este estudo tem como objetivo integrar a evidência a respeito da incerteza face à doença no paciente com doença crônica e seu cuidador familiar, guiados pela teoria da incerteza na doença. Para isso, realizou-se uma síntese integrativa da literatura durante os anos 2007 a 2017. Usaram-se os bancos de dados Medline, Science Direct, Ovid Nursing, Scielo, Scopus, CINAHL e Psycinfo nos idiomas inglês e espanhol. Para realizar a síntese integrativa incluíram-se 46 publicações, com 21 estudos de tipo qualitativo, 19 quantitativo, 2 mistos e 4 revisões. Os estudos realizaram-se, em sua maioria, em pacientes com câncer, doenças neurodegenerativas, falhas orgânicas, falha cardíaca, EPOC e em cuidados paliativos de diferentes doenças. Dentro das principais conclusões do estudo, propõe-se que a pessoa com doença crônica e seu cuidador familiar desenvolvem incerteza na doença crônica devido a falências na educação a respeito da doença e do cuidado, bem como o suporte social que recebem da equipe de saúde e de suas redes de apoio.

Palavras-chave: cuidadores; doença crônica; enfermagem baseada na evidência; ética em enfermagem; incerteza; tomada de decisões 


\section{Introducción}

En 2015, 40 de las 56 millones de muertes mundiales se dieron a causa de las Enfermedades Crónicas No Transmisibles (ECNT) (OMS, 2014), situación que aumentó la proporción de muerte prematura y es un problema para el desarrollo económico de los países (oms, 2015). Sin embargo, el impacto no solo es económico y a nivel de los sistemas de salud, sino en la experiencia vivida de las personas y sus familias, en particular los cuidadores familiares. Situaciones como el trastorno de la cotidianidad, cambio en el autoconcepto (Venning, Eliott, Wilson y Kettler, 2008), síntomas depresivos, carga financiera, familiar y de cuidado (Sautter et al., 2014) son algunos de los impactos vividos, en donde la búsqueda de significado que se construye, en parte sobre la coherencia de los hechos vivenciados, puede incidir en una experiencia menos adversa y en algunos casos positiva, que haga más llevadera la experiencia de vivir con una ECNT (Ballew, Hannum, Gaines, Marx y Parrish, 2012). Cuando se padece una enfermedad crónica, pacientes y cuidadores generan un vínculo estrecho que lleva a que conformen una díada (Chaparro, 2009).

Con frecuencia la vivencia de la enfermedad crónica implica en la díada una dificultad para entender el curso de esta, situación a la que Mishel ha denominado incertidumbre ante la enfermedad (Mishel, 1988). Existen varias situaciones que pueden generar incertidumbre ante la enfermedad en la díada, como son el desconocimiento sobre la enfermedad, su tratamiento, el pronóstico, los síntomas relacionados, la gravedad de la enfermedad (Wolfe-Christensen, Isenberg, Mullins, Carpentier y Almstrom, 2008), entre otras; dadas esas situaciones, es útil disponer de evidencia científica para su abordaje. Sin embargo, brindar cuidado para disminuir la incertidumbre ante la enfermedad en la díada, aplicando la evidencia científica disponible, requiere una estructura conceptual que respalde su intencionalidad, las acciones y la evaluación del cuidado. La incertidumbre existe en situaciones de enfermedad, que son ambiguas, con un alto grado de complejidad e impredecibles. En estos casos está descrita como la inhabilidad para determinar el significado de los eventos relacionados con la enfermedad (Mishel, 1988).

En el cuidado al paciente con enfermedad crónica se deben tomar muchas decisiones relacionadas con la asistencia a estas personas, lo que implica que para garantizar el principio de beneficencia y no maleficencia, este proceso de toma de decisiones debe ser técnicamente sólido. Para esto, el profesional de salud debe tener argumentamos basados no solo en su experiencia, sino también en la evidencia científica, los cuales le permitirán tomar las mejores decisiones atendiendo a las necesidades identificadas en el contexto de la incertidumbre ante la enfermedad.

Por tal motivo, esta revisión de literatura responde al llamado de informar a los profesionales de la salud sobre lo que una gran cantidad de estudios han descrito en el desarrollo de la incertidumbre ante la enfermedad crónica de pacientes y sus cuidadores familiares. Poder realizar una revisión inclusiva y rigurosa favorece que las decisiones se tomen en pro del cuidado del paciente y su familia. Considerando lo anterior y en virtud de la existencia de la teoría propuesta por Mishel (1988), esta revisión tiene como propósito integrar la literatura disponible acerca de la incertidumbre ante la enfermedad en la díada paciente con enfermedad crónica-cuidador familiar, bajo los supuestos de la teoría de la incertidumbre ante la enfermedad.

\section{Materiales y métodos}

Se realizó una revisión sistemática de la literatura de alcance integrativo (Dixon-Woods, Agarwal, Jones, Young y Sutton, 2005) que se llevó a cabo en tres etapas.

Primera etapa: se formuló la pregunta ¿qué reporta la evidencia científica acerca de los conceptos de la teoría de la incertidumbre ante la enfermedad en el caso particular de la ECNT?; la cual dio paso a la búsqueda sistemática de la literatura realizada en las bases de datos PubMed, Science Direct, Ovid Nursing, Scielo, Scopus, CINAHL y Psycinfo. Se usaron los términos MeSH uncertainty, health care provider, health education, social support, cognition, outcomes, nursing y chronic illness combinados con los boleanos AND y OR. Los criterios de 
selección de las fuentes primarias fueron: artículo publicado en revista indexada, en idioma inglés o español, publicado entre 2007 y 2017, que reporte hallazgos relacionados con la pregunta de investigación. La búsqueda se llevó a cabo por los dos autores encontrando inicialmente 3381 artículos, luego de aplicar los criterios de selección se incluyeron 1283 para lectura de títulos y resumen. De estos fueron elegidos 41 artículos y luego de ser leídos completamente se seleccionaron 37. Los artículos que fueron encontrados en idioma español no cumplieron los criterios de inclusión, ya que hacían uso de la teoría en contextos de pacientes críticos o enfermedades agudas.

Segunda etapa: se realizó lectura de las piezas de investigación de acuerdo con lo planteado por Grove, Burns y Gray (2013) con el fin de realizar la selección final de los artículos a incluir en la revisión. Posteriormente se hizo una búsqueda en las referencias de los artículos seleccionados y se agregaron 9 artículos al análisis. En total, se incluyeron 46 estudios entre los años 2000 a 2017.

Tercera etapa: clasificación e integración de los hallazgos de acuerdo con las dimensiones de la teoría de la incertidumbre ante la enfermedad. Esta integración se realizó con ayuda del programa Atlas.Ti 7.0 con licencia de la Universidad Nacional de Colombia.

\section{Resultados}

Sobre los estudios incluidos en la revisión 21 fueron de tipo cualitativo, 19 cuantitativo, 2 mixtos y 4 revisiones. 17 fueron realizados en EE. UU., 8 en Reino Unido, 4 en Australia, 2 en Canadá y los 15 restantes en distintos países, todos estaban en idioma inglés. Dentro del campo de las enfermedades crónicas los estudios se realizaron en su mayoría en cáncer, enfermedades neurodegenerativas, fallos orgánicos, falla cardiaca, Enfermedad pulmonar obstructiva crónica (EPOC) y en cuidados paliativos de distintas enfermedades.

En la figura 2. Se presenta un mapa conceptual que resume los supuestos de la teoría y la interacción entre los conceptos. Así mismo, los resultados del estudio se presentan clasificados de acuerdo a cada uno de los conceptos de la teoría.

\section{Proveedores de estructura para las personas con ECNT y sus cuidadores}

Los proveedores de estructura son aquellos recursos que están disponibles para ayudar al paciente o al cuidador a interpretar y afrontar el marco de estímulos relacionado con la enfermedad. Específicamente cuando se habla de estos proveedores se incluyen

Figura 1. Búsqueda sistemática de literatura y selección de artículos

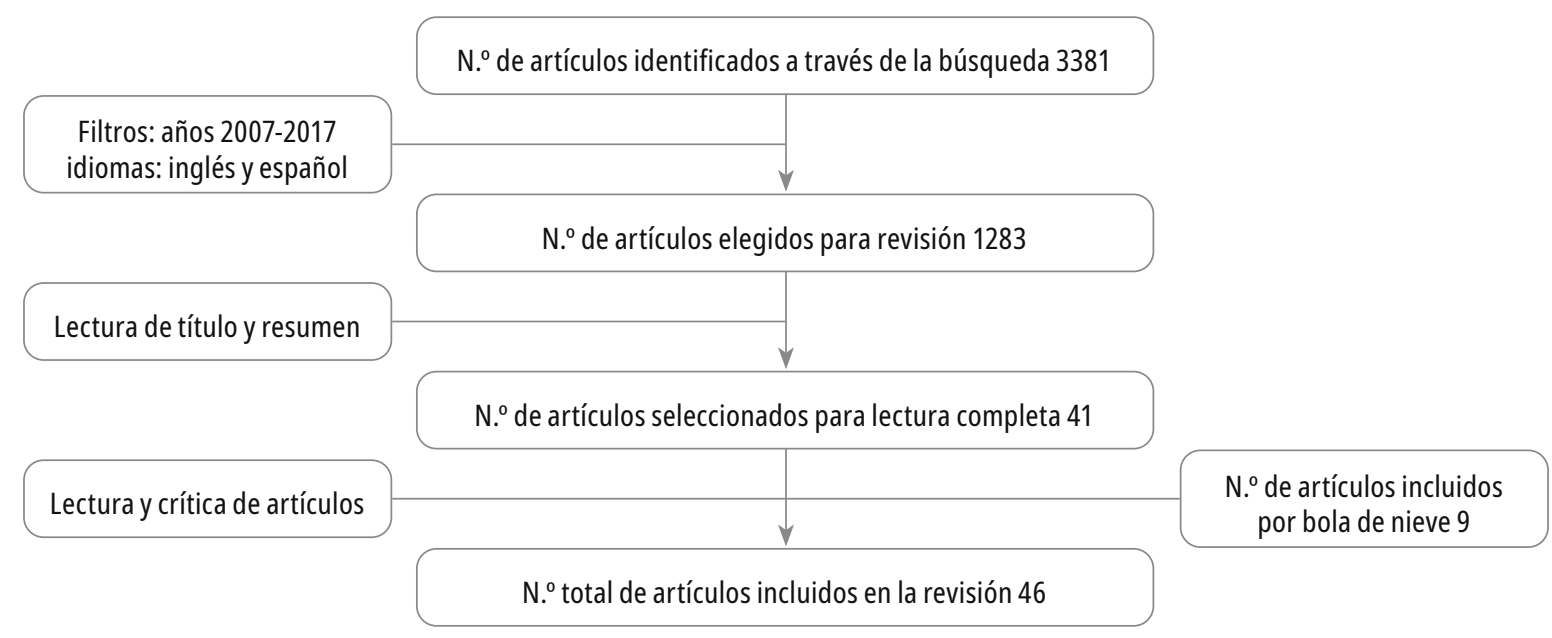

Fuente: datos del estudio. 
Figura 2. Esquema Teoría de la Incertidumbre ante la Enfermedad

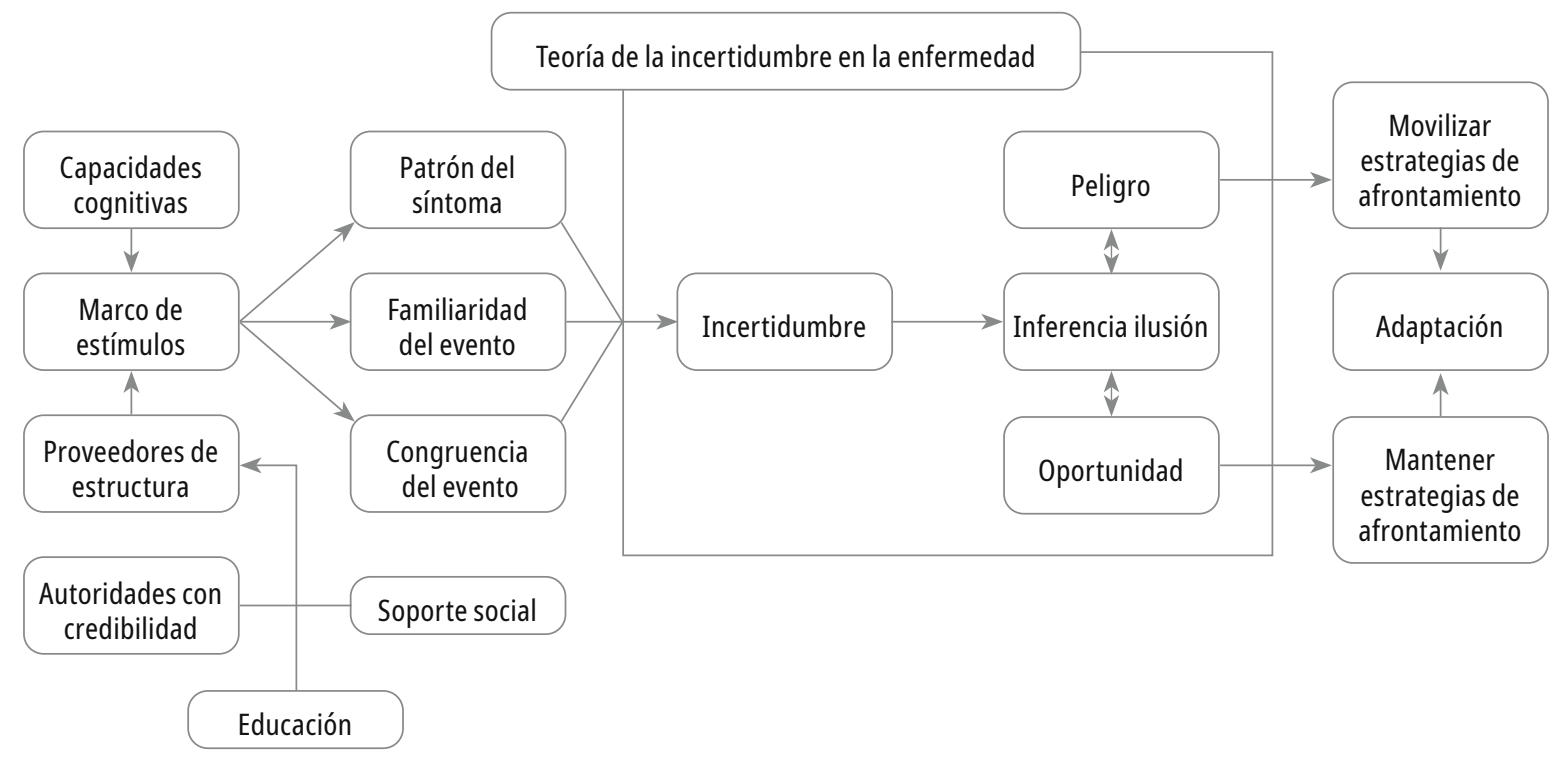

Fuente: adaptado de Mishel, 1988.

tres subdimensiones: la educación, que representa la base de conocimientos de una persona que puede mejorar su capacidad para construir el significado de los eventos relacionados con la enfermedad; el soporte social, que ayuda a disminuir la incertidumbre dando información y asistencia con la evaluación del marco de estímulos de la enfermedad; y, finalmente, se encuentran las autoridades con credibilidad que hacen referencia al grado de confianza que tiene una persona en los profesionales de la salud (Chaparro, 2009; Mishel, Padilla, Grant y Sorenson, 1991). Las anteriores subdimensiones se desarrollan a continuación.

\section{El papel de la educación en la incertidumbre ante la enfermedad crónica no transmisible}

La educación puede ayudar a construir una estructura para el marco de estímulos en la enfermedad, aumentando la base de conocimientos con la que el paciente puede asociar estos eventos y adicionalmente dando un significado y contexto. Para aquellos individuos con un nivel de educación menor a la secundaria, existen niveles más altos de incertidumbre y una percepción de alta complejidad respecto al tratamiento de la enfermedad y el cuidado (Mishel, 1988).

En este sentido, Henson et al. (2016) exploraron la toma de decisiones en las díadas con enfermedad avanzada para acudir a emergencias, encontrando que los aspectos que influencian este proceso dependen del conocimiento y la educación para reconocer la enfermedad y los síntomas. De la misma forma, Sparla et al. (2016) y Lerum, Solbraekke, Holmoy y Frich (2015) concluyen que tanto en pacientes con cáncer de pulmón y enfermedades neurodegenerativas, así como en sus cuidadores, es necesario aumentar la educación para disminuir la percepción del paciente de estar luchando contra sus síntomas y disminuir el miedo de no tener una perspectiva clara sobre el tratamiento (Collier et al., 2017).

Por otra parte, el nivel de educación recibido por las díadas puede disminuir el arrepentimiento en la elección del tratamiento, esto en el caso de pacientes con falla renal $(\mathrm{p}<0.001)$. En estos pacientes, de forma general, la educación influyó disminuyendo el nivel de incertidumbre hasta ocho semanas después de recibir educación $(\mathrm{F}=58.07$, $\mathrm{p}<0.001$ ) (Chiou y Chung, 2012). Adicionalmente, 
Mishel et al. (2003) señalan que los cambios sobre los conocimientos del cáncer de próstata estuvieron regulados por el nivel de educación que tenían los pacientes al respecto $(\mathrm{F}=3.05, \mathrm{p}=.02)$.

Gracias a los hallazgos anteriores se puede decir que un nivel educativo alto y mayor conocimiento sobre la enfermedad y su vivencia podrían disminuir el nivel de incertidumbre ante la enfermedad en pacientes con ECNT y cuidadores.

\section{El papel del soporte social en la incertidumbre ante la enfermedad crónica no transmisible}

Según Mishel (1988), el soporte social sirve para prevenir la incertidumbre en la vivencia de la enfermedad, dando una retroalimentación al significado que tienen estos eventos. Cuando se genera la oportunidad de aclarar las situaciones a través de la discusión y las interacciones con los demás sobre las contingencias sucedidas en el proceso de salud, se ayuda a la persona a formar un esquema cognitivo que integra adecuadamente los conocimientos sobre la enfermedad.

Frente a esto, Sparla et al. (2016) encontraron en pacientes con cáncer de pulmón y en sus cuidadores que los recursos y el soporte ayudaron a un manejo más fácil de la enfermedad. Dentro de estos recursos se encontraron la familia, los cuidadores profesionales y los amigos cercanos. Por otra parte, en el soporte dado por los profesionales de la salud, los familiares refirieron que obtener información acerca de cómo realizar el cuidado del paciente generó un sentimiento de alivio. En concordancia con los resultados anteriores, Hoth et al. (2015) encontraron en pacientes con EPOC que el sentirse criticado por algún miembro de la familia aumenta la ambigüedad respecto a la enfermedad $(b=0.40, p=0.001)$ e influye en el aumento de la incertidumbre.

Borneman et al. (2015) reportaron que más del $65 \%$ de pacientes con cáncer de pulmón y el 50\% de sus cuidadores requerían educación en aspectos relacionados con soporte social y estos requerimientos estaban fundamentados en la necesidad de buscar apoyo de otros que ya vivenciaron la enfermedad. Estos hallazgos son coherentes con los reportados en otros estudios (Hoth et al., 2015; Scott,
Martin, Stone y Brashers, 2011), donde se evidenció que las interacciones sociales de las díadas con grupos de apoyo y reuniones educativas ayudaron a disminuir la incertidumbre de las situaciones relacionadas con la enfermedad. Finalmente, Ang et al. (2015) también encontraron que entre las necesidades más grandes de pacientes con esclerosis lateral amiotrófica se encontraban las de soporte social como recurso de apoyo ante la enfermedad, dado que según estos pacientes es importante escuchar experiencias relacionadas con la enfermedad de otros que ya han pasado por esa situación.

El nivel de soporte social se relaciona con la incertidumbre ante la enfermedad y esta a su vez afecta la calidad de vida de pacientes con ECNT. Somjaivong, Thanasilp, Preechawong y Sloan (2011) exponen que en pacientes con colangiocarcinoma existe una relación entre influencia de los síntomas, el soporte social, la incertidumbre ante la enfermedad y la calidad de vida. Sus resultados evidencian que el soporte social tiene un efecto directo en la calidad de vida del paciente $(\mathrm{y}=0.115, \mathrm{p}<.01)$ $y$ un efecto inverso $(y=-0.275)$ en la incertidumbre ante la enfermedad $(\mathrm{p}<.01)$. De la misma forma, se evidenció que en las mujeres que padecen cáncer de mama y presentan mayor soporte social había menor nivel de incertidumbre ante la enfermedad $(\mathrm{r}=-.288, \mathrm{p}<.002) \quad($ Sammarco y Konecny, 2010; Sammarco, 2001; Neville, 1998).

Los resultados expuestos anteriormente demuestran que existen una asociación entre la incertidumbre ante la enfermedad y el soporte social, $\mathrm{y}$, además, que el soporte social funciona a través de diversas acciones, como dar ayudas tangibles a la persona con ECNT, asesorar y aconsejar, así como compartir experiencias relacionadas con la enfermedad y el tratamiento.

\section{El papel de las autoridades con credibilidad en la aparición de la incertidumbre ante la enfermedad crónica}

Las autoridades con credibilidad hacen referencia al grado de confianza y confidencia que la persona con ECNT o el cuidador tienen en los profesionales de la salud. Usualmente, las personas que vivencian 
la enfermedad buscan una confirmación por parte de profesionales de salud sobre la valoración que estos están haciendo de la experiencia de su enfermedad (Mishel, 1988).

Henson et al. (2016) evidenciaron que uno de los aspectos que regula la toma de decisiones en la enfermedad y la elección de los tratamientos fue la influencia de los profesionales de la salud y los conocimientos impartidos por estos a los pacientes con ECNT. También se evidenció que recibir información por parte de un equipo multidisciplinario redujo la ansiedad relacionada con la aparición de nuevos síntomas y mayor movilización de recursos antes de acudir a emergencias. Igualmente, Aoun, Deas, Howting y Lee (2015) exploraron las necesidades de soporte de cuidadores familiares de pacientes con cáncer cerebral y encontraron que el hecho de tener una oportunidad para discutir con los profesionales de la salud temas relacionados con la enfermedad del paciente fue muy importante para los cuidadores y dio reconocimiento y empoderamiento en la labor del cuidador.

El profesional de la salud, como autoridad con credibilidad, debe, además de instruir y enseñar al cuidador o a la persona con ECNT, tener una escucha activa (Sodowsky, 2012), ya que los cuidadores familiares expresan gran satisfacción con el hecho de poder contar su historia a un profesional de la salud compasivo.

En general, los participantes de los estudios consideraron que los conocimientos de los profesionales de la salud, así como sus habilidades de comunicación y empatía, son cualidades que deben tener las autoridades con credibilidad para poder intervenir en la aparición de la incertidumbre ante la enfermedad.

\section{Capacidades cognitivas de las personas con ECNT y sus cuidadores familiares}

Las capacidades cognitivas dentro del contexto de la incertidumbre en la enfermedad hacen referencia a las habilidades para procesar información de la persona, entre estas se incluyen la atención, percepción, memoria, comprensión y resolución de problemas. Las diversas demandas sobre la capacidad de atención, que suceden con la experiencia de la enfermedad, interrumpen el procesamiento de la información generando así incertidumbre ante la enfermedad. Por ejemplo, esta atención se puede ver reducida por el uso de sedantes, quimioterapia y otros medicamentos (Ballew et al., 2012).

Aunque los estudios de medición de las capacidades cognitivas en pacientes con ECNT son escasos, Kurita, Benthien, Sjogren, Kaasa y Hjermstad (2016) buscaron medir la prevalencia y los predictores del deterioro en la capacidad cognitiva de pacientes con cáncer en cuidados paliativos ( $\mathrm{n}=1477$ ). El estudio mostró que el $28,5 \%$ presentó algún tipo de discapacidad cognitiva y esta aumentó en un $11,4 \%$ con el avance de la enfermedad. Al realizar un análisis de los factores desencadenantes de la discapacidad cognitiva, se encontró que un índice de Karnofsky menor de $70 \%$ en pacientes con cáncer era predictor de discapacidad cognitiva $(\mathrm{OR}=1.6,95 \% \mathrm{CI} 1.0-2.5)$. El estudio también midió aquellos factores predictivos de mejora en el estatus cognitivo y encontró que aquellos pacientes en los cuales su dolor mejoró o se alivió $(\mathrm{p}=.0266)$ e incrementaron su ingesta alimenticia $(\mathrm{p}=.0302)$ disminuyeron su nivel de deterioro cognitivo.

Los resultados anteriores se complementan con los expuestos por Fishbain, Bruns, Disorbio, Lewis y Gao (2010), quienes encontraron que en pacientes con dolor crónico su nivel de incertidumbre ante la enfermedad era mayor que en aquellos pacientes sin dolor; y por los reportados en pacientes con hepatitis C en los cuales un alto grado de fatiga estuvo relacionado con un nivel alto de incertidumbre (Bailey et al., 2010). Por otra parte, el brindar una atención centrada en el paciente y sus síntomas dio como resultado una reducción en el auto-reporte del nivel de incertidumbre ante la enfermedad (Dudas et al., 2013).

Exactamente no se conocen los mecanismos por los cuales existe una relación entre el dolor, la fatiga, la pérdida de apetito; existe la hipótesis de que, por ejemplo, el dolor puede inducir cambios en la utilización de recursos y competir con otros estímulos demandantes de la atención (Mishel, 1988; Mishel, 1990).

De acuerdo con lo anterior, una de las principales recomendaciones al momento de intervenir, 
educar o informar al paciente sobre su estado de salud, sus síntomas, su pronóstico o tratamiento es que esta persona se encuentre libre de dolor o tenga controlados en cierta medida estos síntomas desagradables que compiten con la capacidad de concentración (Mushtaq, Bland y Schaefer, 2011).

\section{El marco de estímulos en las enfermedades crónicas no transmisibles}

Como se ha mencionado anteriormente, las capacidades cognitivas y los proveedores de estructura asisten a la interpretación del marco de estímulos de la enfermedad en las personas con ECNT y en sus cuidadores. Este marco de estímulos hace referencia a la forma, composición y estructura de los estímulos que la persona percibe, y tiene tres componentes: el patrón del síntoma, la familiaridad del evento y la congruencia del evento (Mishel, 1988). A continuación, son desarrollados cada uno de estos.

\section{a. Patrón del síntoma}

Es el grado en el cual los síntomas se presentan con suficiente consistencia para formar un patrón o una configuración. Para evaluar los síntomas, la persona evalúa el número, la intensidad, la frecuencia, la duración y localización, y de acuerdo con esta evaluación se generará mayor o menor incertidumbre (Mishel, 1988).

Dentro de la literatura científica se evidenció en un estudio de cuidadores familiares y pacientes con cáncer (Aoun et al., 2015; Bajwah et al., 2013) que tanto pacientes como cuidadores entendían que la enfermedad limitaba la vida. Sin embargo, pocos tenían un conocimiento completo del mecanismo de funcionamiento de la enfermedad, así como de la forma como la enfermedad se manifestaría en el estado terminal. Este hallazgo refleja cómo la falta de conocimientos dificulta comprender el patrón del síntoma en la enfermedad y genera incertidumbre.

Sobre el patrón del síntoma, también se encontró, en este caso en pacientes con cáncer recién diagnosticados, que estos presentaron hasta treinta y dos síntomas diferentes desde que iniciaron su tratamiento hasta la semana doce después de iniciado el tratamiento y que la intensidad o presencia de estos cambiaba impredeciblemente durante este tiempo; estos hallazgos se compararon con la incertidumbre experimentada, y se encontró que debido a la imprevisibilidad y fluctuación de los síntomas el nivel de incertidumbre se mantuvo en niveles altos desde el inicio del tratamiento hasta después de las 12 semanas (Haisfield-Wolfe et al., 2012). Lo anterior muestra cómo el no entender el patrón de los síntomas en la enfermedad desencadena un nivel alto de incertidumbre.

\section{b. Familiaridad con el evento}

Hace referencia a la naturaleza repetitiva o habitual en un contexto dado. Mientas que el patrón de los síntomas hace referencia a la estructura de sensaciones como las físicas, la familiaridad del evento se refiere a los patrones dentro de un ambiente del cuidado de la salud y se desarrolla al pasar el tiempo y a través de la experiencia en un contexto.

Para desarrollar dicha familiaridad, es necesario generar un mapa mental en la experiencia con el ambiente. Los nuevos eventos en la enfermedad se incluyen en este mapa cognitivo y si estos encajan en la naturaleza del esquema cognitivo del individuo, el evento se evalúa como familiar. En contraposición, el no tener experiencia o enfrentarse a eventos complejos impide desarrollar familiaridad con los eventos (Mishel, 1988).

Para tratar de cuantificar el impacto que tiene la familiaridad con el evento en el desarrollo de la incertidumbre ante la enfermedad, un estudio tomó 158 pacientes con fallo orgánico crónico avanzado y sus cuidadores e identificó las barreras y facilitadores en la comunicación al final de la vida (Van den Heuvel et al., 2016). En los resultados se evidenció que más del $60 \%$ de pacientes como cuidadores refirieron, como una de las barreras más importantes para lograr familiaridad con los eventos de su enfermedad, la comunicación adecuada que se debía dar para afrontar la terminalidad de la enfermedad. Así mismo, Bristowe et al. (2015) encontraron que aquellos pacientes que no recibieron información sobre su pronóstico reportaron gran incertidumbre como resultado de enfrentar la realidad frente a sus suposiciones erróneas sobre la enfermedad. Finalmente, en un estudio con padres de niños con ECNT 
se encontró, sobre la familiaridad con el evento, que una baja habilidad y experiencia en las labores de cuidado del niño, dada por el desconocimiento y falta de instrucción en las mismas, estaba relacionado con un alto nivel de incertidumbre $(\mathrm{p}<0.001)$ (Chaney et al., 2016).

\section{Congruencia del evento}

El último componente del marco de estímulos se refiere a la consistencia entre lo que se espera y lo que se experimenta o vivencia en la enfermedad. Una falta de congruencia entre lo esperado y lo experimentado crea dudas y confusión respecto a la previsibilidad y la estabilidad de la enfermedad (Mishel, 1988).

En relación con el componente de congruencia del evento, cuidadores familiares de pacientes con falla cardíaca identificaron como una de sus principales necesidades que los profesionales de la salud compartieran información con ellos y les dieran una orientación anticipada sobre qué esperar en el futuro (Wordingham, McIlvennan, Dionne-Odom y Swetz, 2016). Este hallazgo, junto con los reportados en otros estudios (Kimbell, Boyd, Kendall, Iredale y Murray, 2015; Ek, Andershed, Sahlberg-Blom y Ternestedt, 2015) demuestran que la falta de información y experiencia provoca inseguridad sobre cómo planear y afrontar el progreso de la enfermedad y el afrontamiento de la muerte.

En esta búsqueda de congruencia, Lillrank (2003) encontró en un grupo de mujeres con diagnóstico de dolor de espalda crónico que los niveles de incertidumbre respecto a la enfermedad fueron muy altos en el transcurso desde que inició su dolor hasta que fueron diagnosticadas correctamente. El esfuerzo que hicieron las participantes por legitimar su experiencia fue parte del proceso de búsqueda de significado que culminó una vez el médico pudo establecer correctamente un diagnóstico que concordaba con su vivencia de la enfermedad.

Por otra parte, según lo reportado en la literatura (Hansen et al., 2012), se sugiere que la educación al paciente se haga de una comunicación sencilla, clara, precisa y entendible. Los pacientes aprecian que los profesionales de la salud den un flujo de información regulada, honesta y objetiva basada en las experiencias de los pacientes, lo que crea una sensación de esperanza real y adaptada a la situación.

Los tres conceptos descritos anteriormente componen el marco de estímulos y se puede generar incertidumbre en cada uno de ellos por separado o en combinación. Cuando la incertidumbre ante la enfermedad existe o aparece, las labores de percepción, reconocimiento y clasificación no se completan adecuadamente, lo que produce que se obstaculice la formación de un esquema cognitivo. Además, para que un evento relacionado con la enfermedad pueda ser clasificado y reconocido este debe tener las características de ser específico, familiar, consistente, completo y limitado en número y en fronteras. Es así que, cuando ese estímulo o evento no tiene alguna de estas características, ocurren errores en el procesamiento cognitivo en la persona y la incertidumbre resulta como un estado predominante (Mishel, 1988; Mishel et al., 1991).

\section{Evaluación de la incertidumbre ante la enfermedad crónica no transmisible}

La persona con ECNT experimenta incertidumbre ante la enfermedad y la evaluación que se haga de esta puede ser tanto positiva, entendiendo la incertidumbre como una oportunidad, como negativa, evaluando esta como una amenaza (Mishel, 1988).

En el contexto de la ECNT la evidencia ha demostrado que los cuidadores evalúan la incertidumbre de forma negativa cuando no tienen las habilidades instrumentales, espirituales o emocionales para llevar a cabo adecuadamente las demandas del cuidado como medicación (Wennman-Larsen y Tishelman, 2002; Moore et al., 2013; Grimmer, Moss y Gill, 2000), cambios de posición, movilizaciones, manejo de síntomas, entre otras (McMillan et al., 2006; Kulkarni et al., 2014; Hasson et al., 2010; Murray et al., 2002). Esta evaluación negativa puede contribuir, entre otras cosas, a generar sobrecarga del cuidador $(\mathrm{r}=.51, \mathrm{p}<0.001)$ y sentimientos de tristeza y depresión (Rabow, Hauser y Adams, 2004; McIlfatrick, 2007).

En estos casos, las estrategias de afrontamiento de la persona enferma o de su cuidador deben ir orientadas a estrategias como la búsqueda de 
información y recursos; y el manejo del afecto, que hace referencia a métodos para lograr esperanza, desenganche y apoyo cognitivo (Mishel, 1988).

Por otra parte, los cuidadores familiares también pueden evaluar la incertidumbre de adquirir este nuevo rol de cuidador como una oportunidad para el crecimiento personal y un fortalecimiento de la relación con su ser querido. Por ejemplo, se encontró que ofrecer seguimiento en el hogar, por parte de un profesional de la salud, favorece el manejo de la medicación, facilita, controla los síntomas, disminuye la incertidumbre, facilita la discusión en torno a los temas del final de la vida y da a los cuidadores una sensación de seguridad y supervisión en las labores del cuidado (Vahidi et al., 2016; Zhou et al., 2012; Hayle, Coventry, Gomm y Caress, 2013). Para personas con cáncer al final de la vida, una evaluación positiva de la incertidumbre puede reflejarse en el restablecimiento de la fe y la esperanza hacia nuevas metas (Holley, Gorawara-Bhat, Dale, Hemmerich y Cox-Hayley, 2009).

Para evaluar la incertidumbre como oportunidad, los métodos de afrontamiento son usados para mantener la incertidumbre en niveles bajos. Como estrategias para prevenir que se altere la incertidumbre como una oportunidad se encuentran la evitación, la postergación selectiva, el reordenamiento de prioridades y la neutralización.

\section{Discusión}

La evidencia empírica revisada en este trabajo indica que la incertidumbre ante la enfermedad es un estado cognitivo en el que las díadas presentan dificultades para comprender y asignar un significado a los eventos relacionados con la enfermedad, debido a desconexiones en la relación entre sus capacidades cognitivas y los recursos ofrecidos por los proveedores de estructura, lo que no permite clarificar un patrón del síntomas o eventos, familiarizarse con ello y además encontrarlos congruentes con lo que se cree que está ocurriendo.

Dentro de los proveedores de estructura en la enfermedad crónica, se resalta que tanto la educación como la interacción con autoridades creíbles y la recepción y adecuada percepción de soporte social (Wennman-Larsen y Tishelman, 2002; Zhou et al., 2012) ayudan a la persona enferma y a su cuidador a contar con la información necesaria para comprender los eventos relacionados con la enfermedad, identificar y anticiparse a los eventos y síntomas (Mishel et al., 1991), buscar ayuda oportuna y eficaz (Lerum et al., 2015); situaciones todas que han mostrado tener efecto sobre la disminución del miedo (Sparla et al., 2016), la ansiedad, la ambigüedad (Hoth et al., 2015; Scott et al., 2011), la carga de cuidado y la imprevisibilidad (Sparla et al., 2016).

Aunque los proveedores de estructura juegan un rol importante en la incertidumbre ante la enfermedad crónica, las capacidades cognitivas de la persona enferma y su cuidador familiar modulan la forma en que las señales se reciben, se interpretan, contextualizan e integran. La literatura muestra que un nivel bajo de escolaridad (Mishel, 1988), el deterioro físico y cognitivo, síntomas descontrolados (Fishbain et al., 2010; Bailey et al., 2010), baja ingesta alimenticia, medicamentos con efectos terapéuticos o colaterales sobre el estado mental (Mishel, 1988), aumentan la incertidumbre ante la enfermedad crónica.

Prepararse para vivir y afrontar todos los eventos previsibles e impredecibles de la enfermedad es una tarea que reviste una alta complejidad, ya que ante cualquier enfermedad, tanto quien la padece como su cuidador siempre guardan la esperanza de recuperación, situación que enfrenta al deseo con la realidad y redunda en incertidumbre ante la enfermedad crónica. En este punto, es necesario comprender el patrón de la enfermedad crónica, e incluso llegar a prever que su curso en muchos casos será imprevisible (Mishel, 1988; Haisfield-Wolfe et al., 2012; Mushtaq et al., 1990). El conocimiento que se tiene acerca de la enfermedad, el contar con apoyo para expresar dudas y sentimientos y la experticia en el cuidado, permiten comprender de mejor manera el día a día de la enfermedad crónica y, por tanto, lograr mayor familiaridad con ella (Sammarco, 2001; Neville, 1998; Chaney et al., 2016), además de construir y aceptar un escenario futuro realista sobre el curso que tomará la enfermedad y los eventos relacionados con ella; en otras palabras, conciliar el deseo y la realidad (Daneault et al., 2016; Tallman, Greenwald, Reidenouer y Pante, 2012). 
Los resultados de esta revisión plantean una serie de implicaciones prácticas. La incertidumbre ante enfermedad percibida por personas con ECNT y sus cuidadores es un factor importante en la determinación de las acciones de cuidado y toma de decisiones respecto a la enfermedad; en consecuencia, las intervenciones pueden centrarse en proporcionar educación sobre la enfermedad, el pronóstico y el tratamiento de la persona con ECNT; así como en aquellos aspectos relacionados con la consecución de soporte social, establecimiento de redes de apoyo, comunicación asertiva y búsqueda activa de información.

Dado que la incertidumbre ante la enfermedad es percibida por muchos cuidadores como la pérdida de control sobre la situación de su ser querido, las intervenciones también podrían centrarse en la adquisición de habilidades instrumentales y el desarrollo de niveles de autonomía apropiados para el manejo de la enfermedad por parte del cuidador. Finalmente, dado el énfasis reciente en modelos de intervención basados en la evidencia (Church, Feinstein, Palmer-Hoffman, Stein y Tranguch; 2014), se plantea como futura necesidad de investigación la evaluación de la eficacia de intervenciones cognitivas que se adecúen al ambiente y condiciones particulares de las personas con distintas ECNT y sus cuidadores familiares y que se estructuren a partir del marco conceptual de la teoría de la incertidumbre ante la enfermedad.

\section{Conclusión}

La díada persona con enfermedad crónica y su cuidador familiar desarrollan incertidumbre ante la enfermedad crónica debido a fallos en la educación y soporte social que se reciben de las redes de apoyo y el equipo de salud, lo que impide que estas sean vistas por la díada como autoridades con credibilidad. Además, el deterioro progresivo y sus repercusiones sobre la cognición de la persona con enfermedad crónica distraen la atención sobre eventos como los síntomas y la imprevisibilidad, lo que afecta la disposición cognitiva para recibir, interpretar, contextualizar, integrar y asignar significado a las señales emitidas por la propia enfermedad. Para comprender y afrontar el patrón de la enfermedad crónica, familiarizarse con él y encontrarlo congruente entre lo que se espera y lo que sucede, es necesaria la unión de condiciones adecuadas en la díada que dependen de aspectos como su nivel educativo, presencia de síntomas, entre otros; y en los proveedores de estructura, que dependen del grado de educación impartida, el vínculo generado con el paciente y cuidador, y su experiencia en el área.

Los resultados del estudio arrojan información importante sobre la importancia que tiene la revelación de la verdad frente a la enfermedad, tanto para el paciente como su cuidador familiar. En este caso, cuando el profesional de la salud educa sobre la enfermedad y su tratamiento, no solo disminuye la incertidumbre ante la enfermedad, sino que favorece el proceso de toma de decisiones en aspectos como la elección de los tratamientos o preferencias de atención en salud. Por otra parte, se sabe que la contextualización de las díadas respecto a la enfermedad alivia síntomas angustiantes, facilita el cuidado de la persona y mejora la calidad de vida. Por esta razón, es imperativo que en la ECNT el cuidado no solo sea técnicamente sólido, sino que involucre la comunicación efectiva y la inclusión de las díadas en todo el proceso, puesto que de esta manera se respetan los principios de la atención con beneficencia y no maleficencia.

\section{Referencias}

Ang, K., Umapathi, T., Tong, J., Ng, J., Tseng, L.J. \& Woo, I.M. (2015). Healthcare Needs of Patients with Amyotrophic Lateral Sclerosis (ALS) in Singapore: A patient-centred qualitative study from multiple perspectives. J Palliat Care, 31(3),150-7.

Aoun, S. M., Deas, K., Howting, D. \& Lee, G. (2015). Exploring the Support Needs of Family Caregivers of Patients with Brain Cancer Using the CSNAT: A Comparative Study with Other Cancer Groups. PLoS One, 10(12), e0145106.

Bailey, D. E., Barroso, J., Muir, A. J., Sloane, R., Richmond, J., McHutchison, J. et al. (2010). Patients with chronic hepatitis $\mathrm{C}$ undergoing watchful waiting: Exploring trajectories of illness uncertainty and fatigue. Res Nurs Health, 33(5), 465-73.

Bajwah, S., Koffman, J., Higginson, I. J., Ross, J. R., Wells, A. U., Birring, S. S. et al. (2013). "I wish I knew more..." the end-of-life planning and information needs for end-stage fibrotic interstitial lung disease: views of 
patients, carers and health professionals. BMJ Support Palliat Care, 3(1), 84-90.

Ballew, S.H., Hannum, S. M., Gaines, J.M., Marx, K.A. \& Parrish, J.M. (2012). The role of spiritual experiences and activities in the relationship between chronic illness and psychological well-being. J Relig Health, 51(4), 1386-96.

Borneman, T., Sun, V., Williams, A.C., Fujinami, R., Del Ferraro, C., Burhenn, P.S. et al. (2015). Support for Patients and Family Caregivers in Lung Cancer: Educational Components of an Interdisciplinary Palliative Care Intervention. J Hosp Palliat Nurs, 17(4), 309-18.

Bristowe, K., Carey, I., Hopper, A., Shouls, S., Prentice, W., Caulkin, R. et al. (2015). Patient and carer experiences of clinical uncertainty and deterioration, in the face of limited reversibility: A comparative observational study of the AMBER care bundle. Palliat Med, 29(9), 797-807.

Chaney, J.M., Gamwell, K.L., Baraldi, A. N., Ramsey, R. R., Cushing, C.C., Mullins, A.J. et al. (2016). Parent Perceptions of Illness Uncertainty and Child Depressive Symptoms in Juvenile Rheumatic Diseases: Examining Caregiver Demand and Parent Distress as Mediators. $J$ Pediatr Psychol, 41(9), 941-51.

Chaparro Díaz, L. (2009). Trascender en un "vínculo especial" de cuidado: el paso de lo evidente a lo intangible. [Tesis doctoral]. Facultad de Enfermería Universidad Nacional de Colombia. Bogotá, Colombia.

Chiou, C.P. \& Chung, Y.C. (2012). Effectiveness of multimedia interactive patient education on knowledge, uncertainty and decision-making in patients with end-stage renal disease. J Clin Nurs, 21(9-10), 1223-31.

Church, D., Feinstein, D., Palmer Hoffman, J., Stein, P. K. \& Tranguch, A. (2014). Empirically supported psychological treatments: the challenge of evaluating clinical innovations. J Nerv Ment Dis, 202(10), 699-709.

Collier, A., Breaden, K., Phillips, J. L., Agar, M., Litster, C., \& Currow, D.C. (2017). Caregivers' perspectives on the use of long term oxygen therapy for the treatment of refractory breathlessness: a qualitative study. J Pain Symptom Manage, 53(1), 33-39.

Daneault, S., Lussier, V., Mongeau, S., Yelle, L., Cote, A., Sicotte, C. et al. (2016). Ultimate journey of the terminally ill: Ways and pathways of hope. Can Fam Physician, 62(8), 648-56.

Dixon-Woods, M., Agarwal, S., Jones, D., Young, B. \& Sutton, A. (2005). Synthesising qualitative and quantitative evidence: A review of possible methods. J Health Serv Res Policy, 10(1), 45-53. http://dx.doi. org/10.1177/135581960501000110
Dudas, K., Olsson, L.E., Wolf, A., Swedberg, K., Taft, C., Schaufelberger, M. et al. (2013). Uncertainty in illness among patients with chronic heart failure is less in person-centred care than in usual care. Eur J Cardiovasc Nurs, 12(6), 521-8.

Ek, K., Andershed, B., Sahlberg-Blom, E. \& Ternestedt, B.M. (2015). “The unpredictable death". The last year of life for patients with advanced COPD: Relatives' stories. Palliat Support Care, 13(5), 1213-22.

Fishbain, D.A., Bruns, D., Disorbio, J.M., Lewis, J.E. \& Gao, J. (2010). Exploration of the illness uncertainty concept in acute and chronic pain patients vs community patients. Pain Med, 11(5), 658-69.

Grimmer, K. A., Moss, J.R. \& Gill, T. K. (2000). Discharge planning quality from the carer perspective. Qual Life Res. Centre for Allied Health Research, University of South Australia, 9(9):1005-13.

Grove, S. K., Burns, N. \& Gray, J.R. (2013). The practice of nursing research appraisal, synthesis and generation of evidence. 7th ed. St. Louis, EE. UU.: Elsevier Inc.

Haisfield-Wolfe, M.E., McGuire, D.B., Soeken, K., Geiger-Brown, J., De Forge, B. \& Suntharalingam, M. (2012). Prevalence and correlates of symptoms and uncertainty in illness among head and neck cancer patients receiving definitive radiation with or without chemotherapy. Support care cancer Off J Multinatl Assoc Support Care Cancer, 20(8), 1885-93.

Hansen, B.S., Rortveit, K., Leiknes, I., Morken, I., Testad, I., Joa, I. et al. (2012). Patient experiences of uncertainty. A synthesis to guide nursing practice and research. $J$ Nurs Manag, 20(2), 266-77.

Hasson, F., Kernohan, W.G., McLaughlin, M., Waldron, M., McLaughlin, D., Chambers, H. et al. (2010). An exploration into the palliative and end-of-life experiences of carers of people with Parkinson's disease. Palliat Med, 24(7), 731-6. Available from: http://pmj. sagepub.com/content/24/7/731.abstract

Hayle, C., Coventry, P. A., Gomm, S. \& Caress, A. L. (2013). Understanding the experience of patients with chronic obstructive pulmonary disease who access specialist palliative care: A qualitative study. Palliat Med, 27(9), 861-8. Available from: http://pmj.sagepub.com/content/27/9/861.abstract

Henson, L. A., Higginson, I. J., Daveson, B. A., Ellis-Smith, C., Koffman, J., Morgan, M. et al. (2016). "I'll be in a safe place": a qualitative study of the decisions taken by people with advanced cancer to seek emergency department care. BMJ Open, 6(11), e012134.

Holley, A. P., Gorawara-Bhat, R., Dale, W., Hemmerich, J. \& Cox-Hayley, D. (2009). Palliative Access Through Care 
at Home: Experiences with an Urban, Geriatric Home Palliative Care Program. J Am Geriatr Soc, 57(10), 192531. Available from: http://dx.doi.org/10.1111/j.15325415.2009.02452.x

Hoth, K. F., Wamboldt, F. S., Ford, D. W., Sandhaus, R. A., Strange, C., Bekelman, D. B. et al. (2015). The social environment and illness uncertainty in chronic obstructive pulmonary disease. Int J Behav Med, 22(2), 223-32.

Kimbell, B., Boyd, K., Kendall, M., Iredale, J. \& Murray, S. A. (2015). Managing uncertainty in advanced liver disease: a qualitative, multiperspective, serial interview study. BMJ Open, 5(11), e009241.

Kulkarni, P., Kulkarni, P., Ghooi, R., Bhatwadekar, M., Thatte, N. \& Anavkar, V. (2014). Stress among Care Givers: The Impact of Nursing a Relative with Cancer. Indian J Palliat Care, 20(1), 31-9.

Kurita, G.P., Benthien, K.S., Sjogren, P., Kaasa, S. \& Hjermstad, M.J. (2017). Identification of the predictors of cognitive impairment in patients with cancer in palliative care: a prospective longitudinal analysis. Support care cancer, 25(3), 941-949.

Lerum, S. V., Solbraekke, K. N., Holmoy, T. \& Frich, J.C. (2015). Unstable terminality: negotiating the meaning of chronicity and terminality in motor neurone disease. Sociol Health Illn, 37(1), 81-96.

Lillrank, A. (2003). Back pain and the resolution of diagnostic uncertainty in illness narratives. Soc Sci Med, 57(6),1045-54.

McIlfatrick, S. (2007). Assessing palliative care needs: views of patients, informal carers and healthcare professionals. J Adv Nurs, 57(1), 77-86. Available from: http://dx.doi.org/10.1111/j.1365-2648.2006.04062.x

McMillan, S.C., Small, B. J., Weitzner, M., Schonwetter, R., Tittle, M., Moody, L. et al. (2006). Impact of coping skills intervention with family caregivers of hospice patients with cancer. Cancer, 106(1), 214-22.

Mishel, M. (1988). Uncertainty in illness. Image J Nurs Sch, 20, 225-32.

Mishel, M., Germino, B., Belyea, M., Stewart, J.L., Bailey, D.E., Mohler, J. et al. (2003). Moderators of an uncertainty management intervention: for men with localized prostate cancer. Nurs Res, 52(2), 89-97.

Mishel, M., Padilla, G., Grant, M. \& Sorenson, D. S. (1991). Uncertainty in Illness Theory. Nurs Res, 40(4), 236240 .

Mishel, M. (1990). Reconceptionalization of the uncertainty in illness theory. J Nurs Scholarsh, 22(4), 256-62. Available from: http://dx.doi.org/10.1111/j.1547-5069.1990. tb00225.x
Moore, G., Collins, A., Brand, C., Gold, M., Lethborg, C., Murphy, M. et al. (2013). Palliative and supportive care needs of patients with high-grade glioma and their carers: a systematic review of qualitative literature. Patient Educ Couns, 91(2), 141-53.

Murray, S. A., Boyd, K., Kendall, M., Worth, A., Benton, T.F. \& Clausen, H. (2002). Dying of lung cancer or cardiac failure: prospective qualitative interview study of patients and their careers in the community. $B M J$ Br Med J, 325(7370), 929-32. Available from: http:// ezproxy.si.unav.es:2048/login?url=http://search.ebscohost.com/login.aspx?direct=true\&AuthType=ip,ur$1 \& \mathrm{db}=\mathrm{asx} \& \mathrm{AN}=7703660 \&$ lang=es\&site=eds-live

Mushtaq, F., Bland, A. R. \& Schaefer, A. (2011). Uncertainty and Cognitive Control. Front Psychol, 2, 249-54. Available from: http://www.ncbi.nlm.nih.gov/pmc/ articles/PMC3184613/

Neville, K. (1998). The relationships among uncertainty, social support, and psychological distress in adolescents recently diagnosed with cancer. J Pediatr Oncol Nurs, 15(1), 37-46.

OMs. (2014). Noncommunicable Diseases country profiles 2014. Ginebra.

OMs. (2015). Noncommunicable Diseases Progress Monitor 2015 [Internet]. wHo. Ginebra: World Health Organization. Available from: http://www.who.int/nmh/ publications/ncd-progress-monitor-2015/en/

Rabow, M. W., Hauser, J. M. \& Adams, J. (2004). Perspectives on care at the close of life. Supporting family caregivers at the end of life: "they don't know what they don't know”. JAMA J Am Med Assoc, 291(4), 483-91.

Sammarco, A. \& Konecny, L. M. (2010). Quality of life, social support, and uncertainty among Latina and Caucasian breast cancer survivors: a comparative study. Oncol Nurs Forum. United States, 37(1), 93-9.

Sammarco, A. (2001). Perceived social support, uncertainty, and quality of life of younger breast cancer survivors. Cancer Nurs, 24(3), 212-9.

Sautter, J.M., Tulsky, J.A., Johnson, K.S., Olsen, M.K., Burton-Chase, A.M., Lindquist, J.H. et al. (2014). Caregiver experience during advanced chronic illness and last year of life. J Am Geriatr Soc, 62(6), 1082-90.

Scott, A. M., Martin, S.C., Stone, A. M. \& Brashers, D.E. (2011). Managing multiple goals in supportive interactions: Using a normative theoretical approach to explain social support as uncertainty management for organ transplant patients. Health Commun, 26(5), 393-403. Available from: http://search.ebscohost. com/login.aspx?direct $=$ true $\& d b=$ edselc $\& A N=e d-$ selc.2-52.0-79961144640\&lang $=$ es\&site $=$ eds-live 
Sodowsky, K. (2012). Spousal Caregiver Narratives and Credible Authority: Uncertainty in Illness of Spousal Caregivers. Qual Rep, 17(18), 1-24. Available from: http://search.ebscohost.com/login.aspx?direct=true\&$\mathrm{db}=$ eric\&AN=EJ978734\&lang=es\&site=eds-live

Somjaivong, B., Thanasilp, S., Preechawong, S. \& Sloan, R. (2011). The influence of symptoms, social support, uncertainty, and coping on health-related quality of life among cholangiocarcinoma patients in northeast Thailand. Cancer Nurs, 34(6), 434-42.

Sparla, A., Flach-Vorgang, S., Villalobos, M., Krug, K., Kamradt, M., Coulibaly, K. et al. (2016). Individual difficulties and resources. A qualitative analysis in patients with advanced lung cancer and their relatives. Patient Prefer Adherence, 10, 2021-9.

Tallman, K., Greenwald, R., Reidenouer, A. \& Pantel, L. (2012). Living with advanced illness: longitudinal study of patient, family, and caregiver needs. Perm J, 16(3), 28-35.

Vahidi, M., Mahdavi, N., Asghari, E., Ebrahimi, H., Eivazi Ziaei, J., Hosseinzadeh, M. et al. (2016). Other Side of Breast Cancer: Factors Associated with Caregiver Burden. Asian Nurs Res, 10(3), 201-6. Available from: http://dx.doi.org/10.1016/j.anr.2016.06.002

Van den Heuvel, L. A., Spruit, M. A., Schols, J. M., Hoving, C., Wouters, E. F. \& Janssen, D. J. (2016). Barriers and facilitators to end-of-life communication in advanced chronic organ failure. Int J Palliat Nurs, 22(5), 222-9.

Venning, A., Eliott, J., Wilson, A. \& Kettler, L. (2008). Understanding young peoples' experience of chronic illness: a systematic review. Int J Evid Based Healthc, 6(3), 321-36.

Wennman-Larsen, A. \& Tishelman, C. (2002). Advanced home care for cancer patients at the end of life: a qualitative study of hopes and expectations of family caregivers. Scand J Caring Sci, 16(3), 240-7. Available from: http://dx. doi.org/10.1046/j.1471-6712.2002.00091.

Wolfe-Christensen, C., Isenberg, J.C., Mullins, L. L., Carpentier, M. Y. \& Almstrom, C. (2008). Objective Versus Subjective Ratings of Asthma Severity: Differential Predictors of Illness Uncertainty and Psychological Distress in College Students With Asthma. Child Heal Care, 37(3), 183-95. Available from: http://dx.doi. org/10.1080/02739610802151514

Wordingham, S.E., McIlvennan, C.K., Dionne-Odom, J. N. \& Swetz, K. M. (2016). Complex Care Options for Patients With Advanced Heart Failure Approaching End of Life. Curr Heart Fail Rep, 13(1), 20-9.

Zhou, M., Holden, L., Bedard, G., Zeng, L., Lam, H., Chu, D. et al. (2012). The utilization of telephone follow-up in the advanced cancer population: a review of the literature. J Comp Eff Res, 1(6), 509-17. 